\title{
Processing, SwitCHING AND COMMUNICATION OF KNOWLEDGE
}

\author{
Michael R. Peterson, Syed V. Ahamed and Sevki S. Erdogan \\ University of Hawaii at Hilo, 200 Kawili Street, Hilo \\ Hawaii 96720 \\ mrp2, sahamed, sevki@hawaii.edu
}

\begin{abstract}
The domain of knowledge 1 is more encompassing than that of wealth and materials. For dealing with utility of knowledge, all factors (its scarcity, its total utility, its marginal utility, specifically its diminishing marginal utility, its utilitarian value, its exchange value, etc.) that influence the evaluation need to be considered. From a communication perspective, knowledge can be traced backward and extrapolated forward, much like scientific parameter(s). From a structural perspective, we propose that the processing of knowledge be based on the most basic and fewest truisms. These truisms are, in turn, based on reality and they permit the characterization of information and knowledge. To this extent, computational processing does not depend on the philosophic writings of earlier economists. However, the truisms are validated from a longer-term philosophic interpretation of how these truisms have survived so that they can be expanded and reused in scientific and computational environments. This approach permits machines to process knowledge based on the content of a particular piece of information and to enhance content, the presentation and the wealth of knowledge that the information communicates.
\end{abstract}

KEYWORDS

Knowledge representation, Knowledge functions, Knowledge and Information Processing.

\section{INTRODUCTION}

The evolution of society is based on the systematic collection, validation, and deployment of gainful knowledge. Knowledge can range from gossip to well-guarded national secrets. Gossip and rumor which have little value are filtered out of the computational processes. On the other hand, knowledge that is rare or unique enters the computational domain to be examined, refined, and enhanced. Knowledge is collected systematically (from the Internet traffic), validated extensively (from the WWW knowledge banks), and deployed widely (from the dictionary of axioms available from the WWW wisdom bases). The true wealth of knowledge (if there is any) must be processed and extracted from the raw format of knowledge in which it was presented. Knowledge processing becomes a precursor to the enrichment of knowledge or the distilling of wisdom.

1 This work is supported by EPSCoR award EPS-0903833 from National Science Foundation to the University of Hawaii. 
Material and monetary wealth has been discussed by Adam Smith and has evolved as a basis for national and international trade and commerce. John Maynard Keynes (1883-1946) and his fiscal policy issues are still held in esteem in monitoring the growth of nations [1]. Unlike monetary wealth, combined information and knowledge ( $I \ll » K)$ has many facets and implications. Whereas the measurement of wealth is scalar and has a numeric measure as the currency value, the wealth of knowledge has more numerous measures. After all, the evolution of society is based on systematic collection, validation, and deployment of gainful knowledge. Knowledge can range from hearsay to well-guarded national secrets. Unfounded information and gossip have only marginal value and such information has no significance. On the other hand, if the knowledge discloses a rare discovery, an invention, or trade secret, then its value is at a premium. If the information has social significance, is rare and is still not disclosed, then the value of that information is high. However, information kept in total secrecy has no value unless it is derogatory or damaging. Even long and extended periods of torture have been justified (questionably) for prisoners of war who supposedly have "information" about the enemy! Rare and damaging information has only blackmail value. For these reasons, the economics and strategy for dealing with knowledge and information need different considerations from those established in typical economics or game theory [2].

A certain commonality exists in the economics of knowledge and traditional macroeconomics. Money that gets stagnant and does not get invested leads to the liquidity trap [1]. The business community refuses to invest and grow because the economic opportunities are too low even though the interest rates may be low. Valuable technological information that does not find its way into production lines remains as paper in patent offices. In a sense, the possibility of an information-rich but stagnant society starts to become real, somewhat like the Japanese society in the 1980s. Valuable knowledge and information (like money) need deployment. Like savings that are invested (Savings = Investment in classical macroeconomic theory), knowledge (Knowledge $=$ Production in knowledge economy) distilled from information needs to be channeled into corporations. Channeling such knowledge into institutions of learning creates a multiplier effect (like that in the national economy) in the ( $I \ll » K)$ domain.

\section{VELOCITY OF FLOW OF KNOWLEDGE}

A certain velocity of flow of knowledge and information $(I \ll » K)$ is necessary for either information or knowledge to be productive. Information that gets too stagnant (like money during liquidity trap conditions) or too fluid (like money during rampant inflationary conditions) loses its potential to be socially valuable. A certain viscosity in the flow of ( $I \ll \gg K$ ), (like money flow) makes the activity rewarding and economically justified. Information that finds no channel(s) for communication has exhausted its life cycle.

A limited commonality also exists in the economics of information and traditional microeconomics. The value of information and knowledge ( $I \ll » K)$ that is transacted becomes comparable to the value of goods or assets that are transacted. However, ( $I \ll » K)$ does not get depleted like goods or assets that are physically exchanged. The depletion of the value of ( $I$ «» $K$ ) follows an exponential decay rather than a sudden change. The rate of decay can be quite sudden (high exponent) for some types of ( $I \ll \gg K$ ), (e.g., weapons and warfare technologies) compared to others (e.g., educational or medical technologies). The sharing of ( $I \ll » K)$ may bring down the value as an exponential decay but it still retains some utility for both parties. Both parties benefit from the economic rewards yet retain the wealth of information. Monetary and material wealth that is shared loses value and utility simultaneously. The value of ( $I \ll » K)$ varies with server - client 
relationships. Conflictive and cooperative roles are both feasible, thus altering the laws of economics of knowledge and information.

Mainly, ( $I \ll » K$ ) that has social, financial, social, ethical, or moral implication is a resource that is not as immediately exhaustible as monetary or materialistic wealth. Like any other resource, ( $I$ «» $K$ ) can be accumulated, enhanced, stored, or even squandered; however, this resource has special properties. The enhancement of $\left(I_{\ll » K} K\right)$ is a mental/machine activity differing from the enhancement of material wealth, which is a production/robotic activity. For the differences cited above, $(I \ll » K)$, "objects" are treated as hyperdimensional objects that follow the laws of processing but are not quite aligned with the processing of numbers, scalars (such as currency values), or text. Modern computers are capable of processing vectors and graphical objects. Current software packages that handle complex number $(x+i y)$, two dimensional space for electrical engineers and mathematicians perform as smoothly as the software packages that handle threedimensional $(X, Y, Z)$ space for graphics designers and movie makers. In dealing with the $(I \ll » K)$, "objects", special compilers are necessary. Such compilers should perform lexical, syntactic, and semantic analyses of information objects that can identify other information objects and relate themselves to the newly found objects by variable and adaptive role-based linkages. A recursive compiler can handle such a scenario.

The processing of graphics entities [3] starts to assume the initial flavor of the processing of the information objects. Some of the steps suggested in this paper are initial and rudimentary, but they can be modified and enhanced ${ }^{2}$ to suit different types of information object(s) and their interactions. Processing of information objects depends on the application. On the one hand, mechanical and routine transactions of information objects are akin to data processing in banking and commerce. On the other hand, when information has human and social implications, then a new software layer that emulates human processes (such as love, hate, needs, feelings, education, counseling) becomes necessary. Generally, human interactions follow an underlying economic framework of the exchange of resources. On a very short-term basis, the marginal utility theory [4] starts to unfold in most transactions. Perceived fairness and valuation are of essence in most cases. In dealing with information, most humans follow a fairness and value judgment analysis unless it is willfully transgressed.

The rational component of human processes follows simple programming approaches. The emotional component is tackled by suggesting (and adapting) a series of statistical paths ranging from common to rare reactions. Such reactions are documented in the knowledge bases around the world, and steps are adapted in neural networks. In such instances, the machine-generated resolution of information can be superior to all-human solution since machines can evaluate every type of emotional response in every culture and can suggest a customized response closer to the tastes of the humans involved.

While machines are communicating or exchanging information, they strictly abide by the $\mathrm{I} / \mathrm{O}$ commands of humans or of the basic core operating system. While human beings process information, the value and worth of the information are initially assessed, and modified by learning, clarification and negotiation. While machines are processing information, the information processing units ${ }^{3}$ (IPUs) alter the structural relationships between objects and objects, objects and

\footnotetext{
${ }^{2}$ For algebraic operations (multiply, divide, matrix, etc.) for complex numbers, the development of software routines followed much later after the assembly level programs for processing real numbers.

3 Information processing and knowledge processing are used interchangeably in this paper since the forward processing (distilling) of information leads to knowledge and the backward processing (parsing) of
} 
their attributes, and relationships between object $\mathrm{X}$ attributes with object $\mathrm{Y}$ attributes. The scenario is depicted in Figure 1. The alteration and redistribution of relationships is not altogether random (unless it is the last resort).

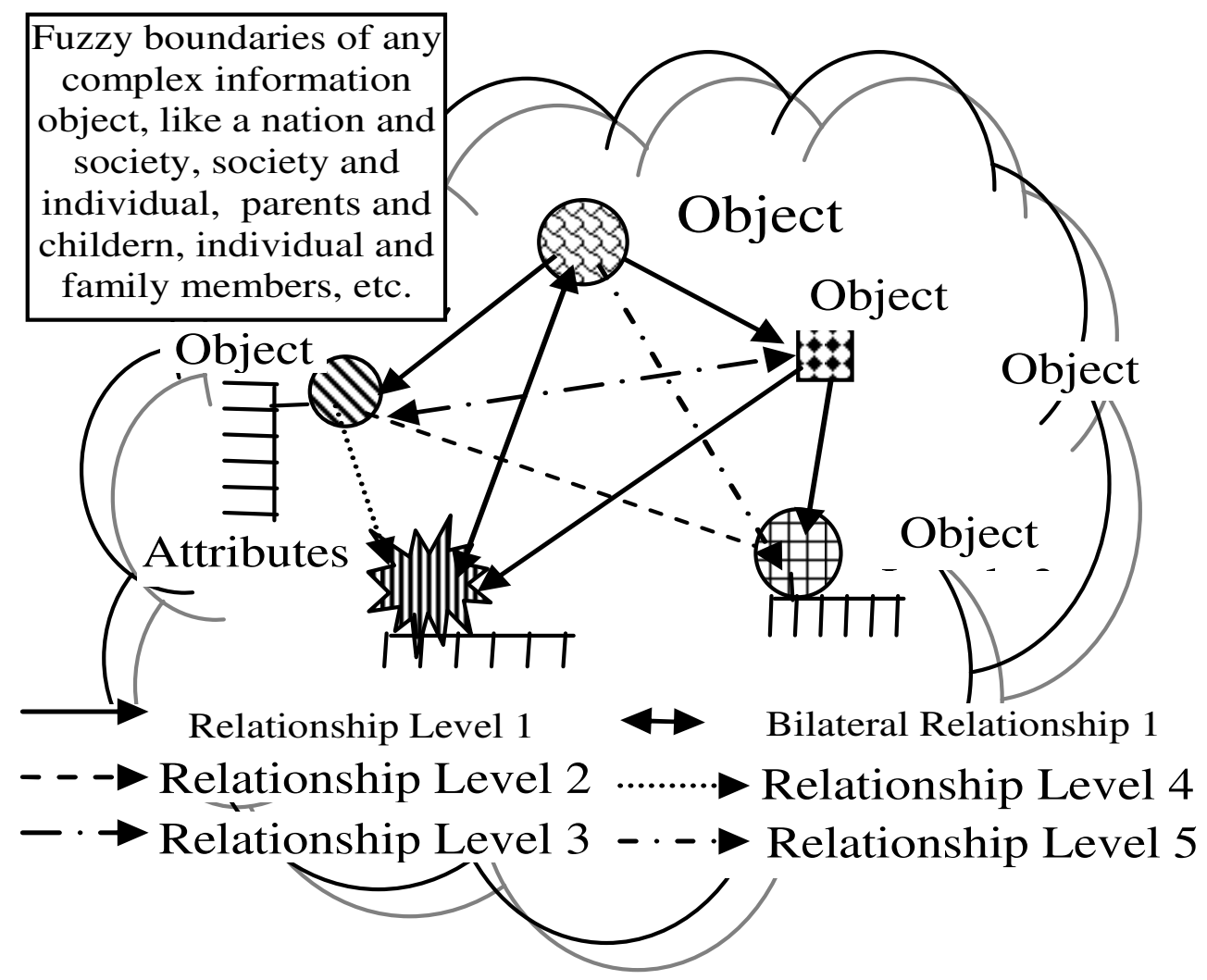

Figure 1 Representation of a complex information/knowledge object with five lower-level information objects. Strong, weak, casual, unilateral, and bilateral relationships are shown.

Instead, they are based on laws of probabilities as to which of the relationships are most common and likely to form secure bonds (e.g., information about automobiles and information about octane values; or information about hang gliders and information about wing span of birds). In the process, the machines also investigate unusual and uncommon relationships (e.g., information about the design of hang gliders for Australian coasts and information about the design of hang gliders for Scandinavian coasts), giving rise to novel and unique information, knowledge, or scientific principles (if any).

Machines have an advantage in processing vast amounts of information quickly and accurately. The incremental changes in information are tallied to the incremental changes in external conditions to optimize and predict the information for a given set of new conditions. Incremental changes over any of the parameters (such as time, attributes, or environmental conditions) are

knowledge leads to information. The same machine may be able to process in either direction, perhaps by changing its control memory chip sets. At this stage, it is premature to speak specifically to the many possibilities that still lie ahead. 
accurately tracked and labeled. Processing the key information object(s) that forms the nucleus (nuclei) of the raw information and then reconstituting the information object(s) identifies opportunities for possibly new and valuable information, knowledge, or scientific principles. This is the fundamental clue to crossing from the information mode to the knowledge mode.

Unlike monetary wealth and the wealth of nations [5] that are depleted, the wealth of information is shared. Unlike monetary wealth, information ( $I \ll \gg K$ ) has significantly different attributes. Whereas universal and numerical values can be assigned to monetary wealth, information has overlapping qualities and fuzzy parameters to transact information.

Complexity theory [6] starts to resemble knowledge theory because of the highly variable nature of $(I \ll » K)$ "objects" and their interrelationships. Most of the precepts of complexity theory become applicable when dealing with information and knowledge. However, in dealing with $(I \ll » K)$, we limit the processing to a confined number of objects that do not make the information processing chaotic. The self-contained structure is prioritized with statistically weighted relationships between the objects that are considered valid for the processing of ( $I \gg K)$ "objects." In addition, the limitations of the computer system (accuracy, memory size, speed, and possible switching capability) define the size of the "body of knowledge" (or the "complex initial object") that the computers will handle.

The knowledge processing system filters out any "objects" that are likely to cause chaotic and unstable oscillations in the processing. It refuses to process inconsistent information, much like computers that refuse garbled data. During the execution phase, irrational requests to process information are terminated and the error condition is intimated, just like computers that refuse to execute impossible numeric operations. Unlike complexity theory, the knowledge theory will perform legitimate functions on objects for which some earlier statistical information is available on world-wide knowledge banks. If the extent of information is too restrictive, the learn mode [7] is invoked to build a knowledge base for the unknown object. The machine guards itself from being drawn into an execution mode that ends in catastrophe by establishing non circular forward and backward pointers. Even though recursion is permitted, the depth of recursion is made consistent with the machine capacity. Rationality is given higher priority than the task of execution of a knowledge program. These bounds of rationality contain the fuzzy bounds of knowledge that is under process. To this extent, the machine regains its own stable operating condition, just as a human being would attempt to do. Thus, overall knowledge processing systems have fair a chance of solving complex knowledge problems that human beings by themselves cannot attempt.

The knowledge processing system limits the size of the body of knowledge processed by a quantitative measure of the capacity of the machine in relation to the requirement of "complex initial object." No such limitation is imposed in complexity theory. For this reason, knowledge theory is based on the computer systems that will attempt to solve a knowledge problem. Knowledge theory is a valid tool in initially formulating the problem and becoming strategic in its solution. The system resource expended to change the status of information and knowledge (see P3 in Section 4) during the course of the solution of the problem will be (in most instances) the bottleneck. In essence, complexity theory is an open-ended theory, but knowledge theory works in the context of machines having discrete (binary or hyperspace) representations, limited in their memory, I/O, switching capacities, and speed of operation.

To this extent, knowledge theory is like information theory that works in most non-chaotic but extremely noisy environments. Knowledge theory does not violate any of the principles (such as auto-organization, edge of chaos, power of connections, circular causality, try\&learn, and ologrammatic principle) set forth by complexity theory. To some extent, auto-organization and 
try\&learn are based on the survey of the world-wide knowledge bases on the Internet to find out how other complex knowledge objects have accomplished auto-organization and adaptation. To this extent, quantification within knowledge theory (like that within information theory) becomes totally feasible.

Shared information loses value at a relatively low rate. Whereas there is a suggestion of strict zerosum game [2] in transacting the wealth of nations and individuals, there is an impression of elastic zero-sum game as two parties share knowledge and information. Wealth (i.e., all the utilities combined together) and value rather than price of information are only perceived at the time of sharing information. The sale price of a commodity or an asset can only arise in a free-market environment. The price for sharing information is perceived between buyer and seller and not determined by market forces. Sometimes the value of information in a document, a book, or a scripture far exceeds the price of the book and sometimes the converse can be the case.

In the knowledge domain, an approximation for the scarcity, value and life of the information is feasible. Along the scarcity, value, and lifetime (three dimensional curve) five coordinates points can be readily identified: (1) totally unshared and secret information has no value and indeterminate life, (2) guarded information has high value and relatively long life, (3) information shared with a select clientele has highest value until it starts to leak and slowly erodes in value, (4) media information has a media price and short life, and finally (5) gossip and trivia has junk value and dissipates without a trail. The value of information in a socioeconomic setting has at least three additional dimensions, the truth contained, the elegance or appeal conveyed, and the social benefit that can be derived from the information.

To deal with the complex nature of information from a computational and processing perspective, we propose four (truism, philosophic, scientific, and economic) dimensions or senses, shown in Figure 2, over which information can be characterized. In dealing with information as an object, truism of all information objects (not their content) states the truth (as well as it is known) about the entire object class. Similarly, philosophic characterization of all information objects (not their content) states the philosophic nature (as well as it is known) about the entire object class, and so on.

Processing an information object can alter its four characteristics (T, P, S, E). In fact, constructive processing will make marginal information (objects) into significant information (objects), if there is any significance. Worthless information is filtered out from any scientific knowledge processing 4 . The process can be deduce, interpret, derive, systematize, analogize, categorize, conceptualize, rationalize, and generalize or any other process that has a scientific basis.

In order to initiate the information processing to search out new information or new knowledge from vast amounts of data, three steps are proposed: observation of reality, philosophic validation, and scientific principles that can be generalized and deployed elsewhere. The observation of reality is fundamental to all sciences. Since information has illusive boundaries and flexible formats, the concept resides in the content of information and goes deeper than a statement or representation of information. In a sense, information is the water that can be poured into any vessel. The water is a real information object with its own properties and the vessel is the secondary information object. Together, they form a (partially) stable object group.

\footnotetext{
4 Most compilers permit programs (program-objects) from proceeding to the execution phase only if they are free of all syntactic, all semantic and all linkage errors. In a similar vein, information that is inherently false, malicious, or laden with pornography will not gain access to information-object processing systems.
} 

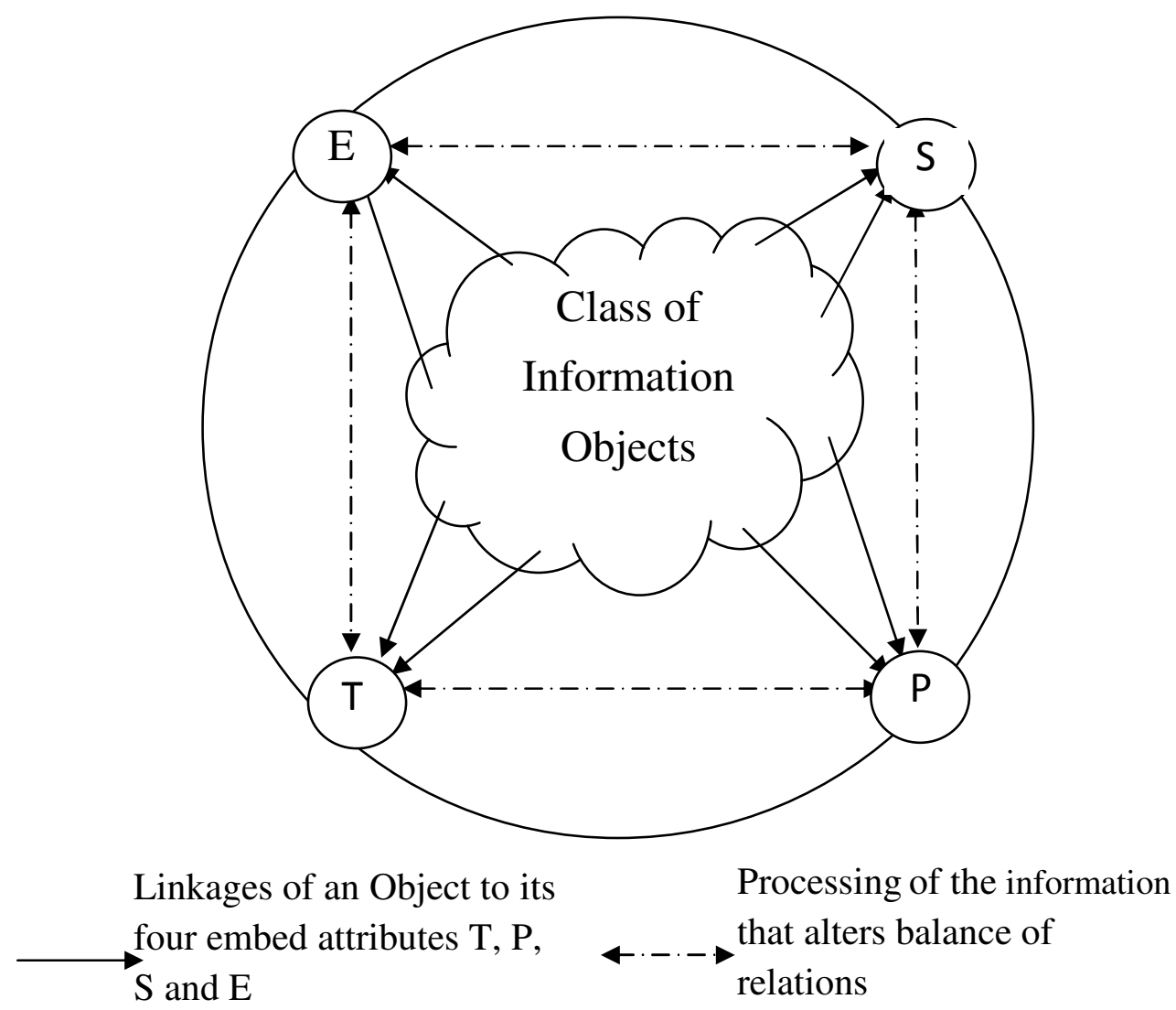

Figure 2 Representation of the class of knowledge and information objects with four characteristics: truism $(\mathrm{T})$, philosophic $(\mathrm{P})$, scientific $(\mathrm{S})$, and economic $(\mathrm{E})$ nature of knowledge.

The philosophic validation is necessary to provide a long-term continuity and stability of information, such that any inference/scientific conclusion can be drawn. To continue with the earlier example, if the water is poured into a vessel carved out of ice, there will be neither water nor ice over a long enough period ${ }^{5}$ of contemplation to form a stable object group (i.e., water in a vessel). It becomes necessary to probe the wealth of knowledge and information (water) in society (vessel) to validate the reality as a stable and dependable basis.

\section{TRUISMS IN KNOWLEDGE DOMAIN}

Observation of reality over long periods leads to generality or truism. In dealing with Knowledge, three notions are suggested:

T1. Knowledge has a life cycle.

T2. Knowledge can be altered, but any alteration of knowledge needs expenditure of energy.

T3. Knowledge has impact.

${ }^{5}$ Unless the situation is adiabatic at $32^{\circ} \mathrm{F}$, which becomes too specific to draw any general conclusions. 
International Journal of Managing Information Technology (IJMIT) Vol.2, No.3, August 2010

The list is short and other dependent truisms can be derived from the three listed above. The truism layer is shown at the top of Figure 3. The T1 to T3 list is kept deliberately short with the hope that the list of derived scientific principles will also be elementary and short. This would reduce the basic operations that a computer system will have to perform while processing knowledge.

\section{PHILOSOPHIC VALIDATION OF KNOWLEDGE}

Only four philosophic validations (P1-P4) are suggested and the list is deliberately kept short to reduce the instruction set for the machine to process knowledge. It is depicted as the middle layer in Figure 3. Based on T1, the justified philosophic validation (at this time) is as follows:

P1. Knowledge is timely or obsolete and it can change its characteristics over time. Typically, human or machine processing changes the derived knowledge. It ranges from mere gossip to a scientific principle or an equation in physics. When the linkages to the raw knowledge are not retained, the processed knowledge may assume the identity of a new knowledge object. Hence the concept of Aristotle's beginning, middle, and end becomes fuzzy in the knowledge domain. We refer to this particular validation as $\mathrm{P} 1$.

Based on T2, two philosophic validations (P2 and P3) are feasible and presented separately because the implications are different.

P2. Boundaries of knowledge and information are vague and fuzzy. Returning to the example of water in a vessel, information is blended in the human perception. Much like the features of beauty that lie in the eye of the beholder, the boundaries of knowledge lie in the mind of the receptor. Human perception becomes a fading memory (or a leaky bucket) to hold knowledge (water). When a machine receives knowledge, the knowledge objects, their structure, and their relationships are analyzed and stored with the timeline for that specific "body" of knowledge. In a sense, the "knowledge compiler" performs a lexical, syntactic, semantic, and timeline analysis on "knowledge inputs" and identifies the knowledge objects, their structures, and their relationships.

Implication $\mathrm{P} 3$ based on $\mathrm{T} 2$ is

P3. Knowledge has three qualitative features; truth contained, social value conveyed, and the inherent elegance in content in variable proportions. Knowledge can also have the opposite features (falsehood, social malice, and ugliness) in variable proportions. An equally important principle is that the change of status of knowledge implies an effort (equivalent to force) to bring about the change sustained over the displacement of the status (thus invoking a concept of psychological or social energy or the deployment of resources).

To fall back on the example of water and the vessel, if the water carries three partially dissolved solutes (sugar, sweetener, and honey), then the viscosity changes thus altering the fluid mechanics and the concentration levels in different sections of the vessel. Furthermore, any alteration of the concentration level, after an equilibrium condition is reached, needs energy for the change (such as stirring, shaking, vibrating, or adding more water). The scientific basis for predicting the concentration contours becomes quite complex and even unpredictable (like the weather). However, when a machine has a basis of estimating the truth (sugar), the social value (sweetener), and the elegance (honey) independently (based on statistical sampling of other knowledge objects 
International Journal of Managing Information Technology (IJMIT) Vol.2, No.3, August 2010

and their relationships), then the raw/processed knowledge can be scientifically evaluated with appropriate confidence levels.

Based on $\mathrm{T} 3$, the validation for $\mathrm{P} 4$ is stated as follows,

P4. Sharing of knowledge can bring rewards or retributions in any variable proportion. This particular implication carries little impact in the scientific domain but becomes significant in the social and economic domains. In the socioeconomic realm, it is generally accepted practice to exchange items of similar value (including knowledge, patents, techniques, and ideas). It is also frequent to find the extent of damage inflicted as retribution. In the knowledge domain, litigation and penalties are imposed when negative knowledge and bad publicity are purposely circulated.

\section{SCIENTIFIC PRINCIPLES IN KNOWLEDGE DOMAIN}

Five scientific principles (S1-S5) are derived from the four philosophic validations (P1-P4). The first principle, S1, results from P1 and is stated as follows.

S1. It is implied that knowledge is dynamic. At any instant, knowledge can be segmented (differentiated with respect to time), encoded, communicated, corrected, interpolated, extrapolated, restored and even reconstituted. Knowledge can be grouped (i.e., integrated over time), independently or with current or past knowledge object(s). If knowledge objects are treated as dynamic and continuous in the time domain, then differentiation and integration become possible. The analog and closed-form operations are irrelevant, but finite and eventdriven changes are sensed from information and knowledge bases. For instance, every scientific meeting or conference adds or subtracts from the collective knowledge base of a community. Human beings and/or machines can process new knowledge-objects continuously. When finite changes are necessary then the commitment of resources becomes essential. Hence the concept of (expected) incremental or marginal costs are evaluated and equated to the (expected) incremental or marginal benefit that is gained.

The second principle, $\mathrm{S} 2$, is derived as an extension of $\mathrm{P} 2$ and is stated as follows:

S2. Boundaries of knowledge and information are vague and fuzzy. Such boundaries are also flexible and can be made inclusive or exclusive of other knowledge objects. Single knowledge object exist as topological structures, "graphs," and/or graphical patterns. Groups of information objects also have structure, graphs, and/or patterns of relationships within the information objects and/or their attributes. Structures, graphs, and patterns (see Figure 1) can have scientific implications for stability. In the domain of knowledge, the knowledge objects need reasonable bonds to remain existent for a period. Insecure bonds between objects only results in short-lived rumors and gossip.

The third principle S3 also results as an extension of $\mathrm{P} 2$ and stated as follows:

S3. A knowledge object can be processed, corrected, recombined, merged, fragmented, and restructured by itself or in conjunction with other knowledge objects. It (they) can also be segmented (differentiated or partially differentiated with respect to other knowledge objects or attributes), grouped (integrated), interpolated, extrapolated, rationalized, and restored in object or attribute space. 


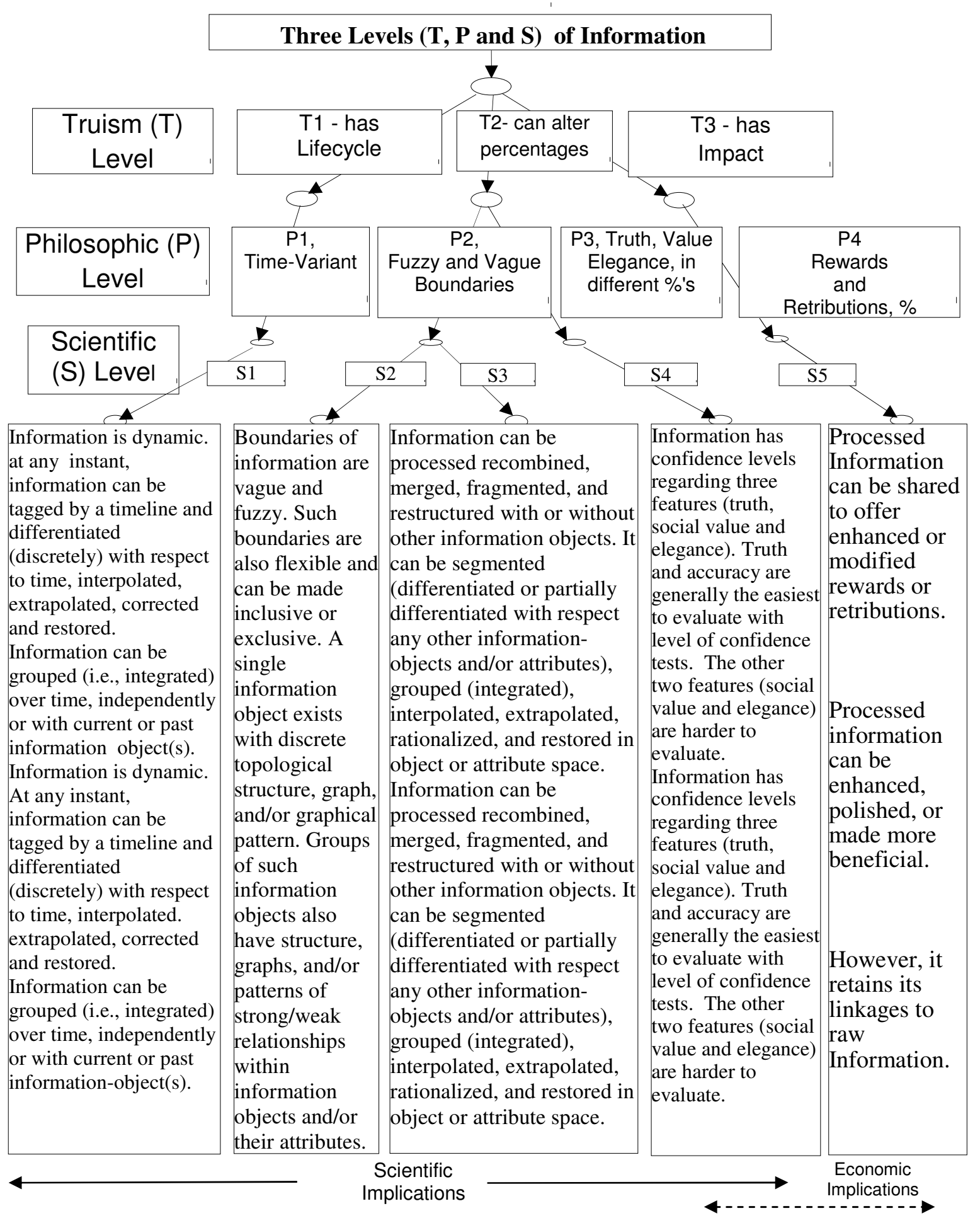

Figure 3 Three (T, P and S) levels of knowledge processing. The S Level is consistent with truism and philosophic handling of knowledge-objects, but the scientific principles derived as the third level (S) offer mathematical tools and techniques to deal with knowledge-objects 
International Journal of Managing Information Technology (IJMIT) Vol.2, No.3, August 2010

The basic tools of (discrete) mathematics become applicable in dealing with the continuity of knowledge over time and the continuity of (structural relationships or discrete) contours with respect to other objects or their attributes.

The fourth principle S4 results from P3 and stated as follows.

S4. Knowledge has confidence levels regarding three features (truth, social value and elegance). Truth and accuracy are generally the easiest to evaluate in context to other similar single knowledge-objects or other multiple knowledge-objects with level of confidence tests. Generally, (local and global) knowledge bases that contain knowledge about similar objects can provide a basis for confidence tests. The other two features (social value and elegance) become harder to evaluate.

The fifth principle S5 results from P4 and is stated as follows:

S5. Processed knowledge can be shared to offer enhanced or modified rewards or retributions. Processed knowledge retains its linkages to raw knowledge. Human processing of knowledge has taken a firm hold in society. Transitory knowledge processed by the human mind is dispersed as conversation. Knowledge that is more important is documented and retained for further reference. In the realm of processing by intelligent machines or systems, knowledge can provide more value (truth, social significance, or elegance) in the processed mode, especially if the processing is done on a scientific basis by following principles (S1-S4). For example, segmentation and recombination offer a slightly different form of truth (that is equally valid) as the original truth. Similarly, mere rearranging of the words can sometimes make a hidden context or idea more apparent and so on. From a computational perspective, simple differentiation tests (i.e., event analysis and correlation studies) can reveal the more sensitive knowledge objects with a complex knowledge structure.

\section{Processing AND SWitching OF KNOWLEDge}

\subsection{KNOWLEDGE PROCESSING FRAMEWORK}

We illustrate the configuration of a typical knowledge processing unit (KPU) and a single processor knowledge processing system (KPS) in Figure 4. The organization of object and instruction registers and caches is presented for a knowledge processor capable of handling each knowledge binary level instruction. From an implementation perspective, the configuration depicted in Figures 5 and 6 with multiple processors, are more desirable. Additional architectures handling, for example, single-process single-object (SPSO) systems are discussed in [8].

In the single-processor environments, communication and the switching can be combined into one hardware unit. In the extreme case, it can look like a disk controller that channels the access to certain track-sector addresses when certain subject matters are being pursued. This simplification is feasible because of the reduced requirements placed on the switching unit of the KPS. This module provides a communication path during the allocated time interval between the only processor (or memory) in the administrative module (accomplishing only one individual knowledge-oriented "task") and the knowledge module. To some extent, the simplification is comparable to shrinking an entire switching system to a single-party PBX. All the switching takes place on the knowledge module side. 


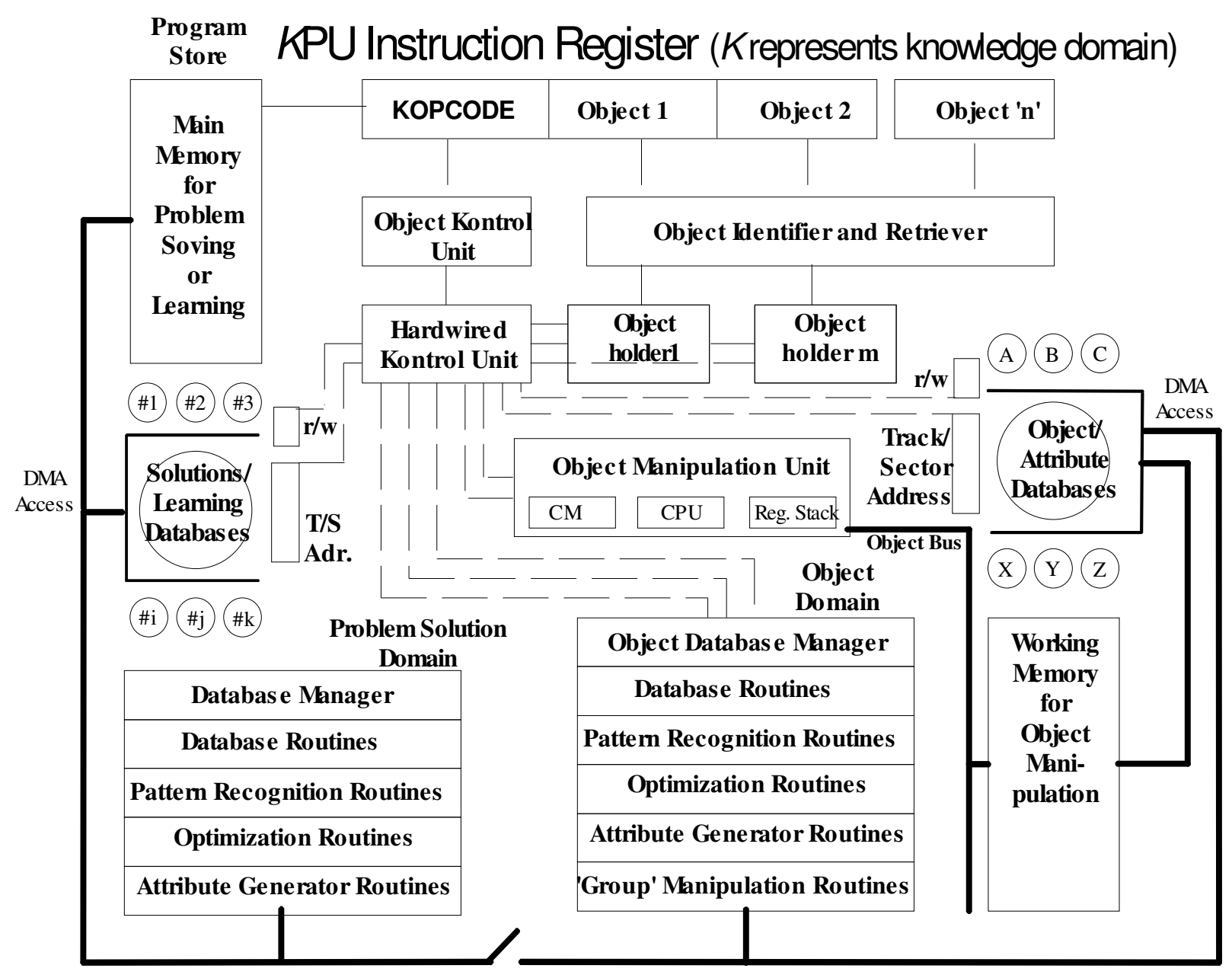

Switch 1, Open for Execution Mbde for Knowledge Domain Problem Soving; Clos ed for Learning Mbde.

The Learning programs 'process' the existing solutions and are able to extract Objects, Groups, Relationships, Opcodes, Group Operators, Modules, Strategies, Optimization Me thodologies from existing solutions and store them in Object and corresponding databases. The architecture permits the KPU to catalog a new object in relation to existing objects and generate/modify existing pointers to and from new objects.

Figure 4 Knowledge processing unit (KPU) architecture based on the knowledge instructions being able to process objects embedded in a given body of knowledge or module of information. The unit can serve to execute knowledge instruction to alter the flavor of information or it can learn the attributes of objects and interrelations. 
The administrative functions assure error free execution of knowledge programs. These functions accomplish the compilation of the steps invoked in the solution of the knowledge-oriented problems. We can also foresee that the steps invoked in the solution of intricate problems will depend on the results of the prior steps, with a "compile-as-you-go" approach being necessary. Sometimes communication engineers also use this approach during the implementation of network algorithms (such as path routing, use-dependent path assignment, fault tolerance, etc.)

In addition, this administrative unit (Figure 5) has the support of a disk-resident "knowledge profile" of the knowledge module. In essence, the profile provides an initial checkpoint when the users invoke operations pertaining to the knowledge stored in the knowledge module(s). It is somewhat similar to the card-file index that older libraries once maintained to facilitate users in finding the exact location of the textbook containing the subject matter they were pursuing. The profile also verifies that users ask questions of the system that are consistent with the knowledge contained therein.

For example, it identifies what action 6 may be applicable to what subject matter (such as, polymers, petrochemicals, Maxwell's equations, and satellite systems). In a simplistic case where the machine is geared toward high school teaching, the profile can be preshrunk from the more elaborate profile of the machine that is oriented to a graduate research center where the subject matters are processed.

This customizing of the disk-resident profile is similar to the editing of the table of contents for textbooks covering the same subject for high school or college use. From the perspective of the computer software designers, this customizing is similar to the selection of different compilers for different hardware modules (e.g., co-processors, array processors, and A to D converters) or software library routines (e.g., trigonometric, hyperbolic, or exponential).

The administrative module may also be invoked to modify and manage the knowledge bases (a function similar to the function of the service management systems in intelligent networks) and the corresponding updating of the knowledge profile. Additional security checks of the access and updating of users, plus their use of the knowledge modules, may also be incorporated here. The compiled and executable task-oriented program may also be made disk-resident or handled in the main memory of the KPS. These routine housekeeping functions are also managed by the AM.

Figure 5 depicts the four basic modules of the KPS and one of the ways of interconnecting the components. In this arrangement, the switching module (SM) is located between the communication module (CM) and the knowledge bases (KBs). If the various $\mathrm{KBs}$ are arranged in some well-defined hierarchical format (such as the Dewey Decimal System, [8] or the Library of Congress classification [9]), then a network configuration (star, bus, ring, dropped bus, etc.) can be used to retain the access methodology in and out of vast databases. Under these conditions, one BISDN (broadband ISDN) link or one access point may be provided between the switching module (SM) and the knowledgebase (KBs).

With its resident operating system, the administrative module (AM) also dynamically allocates resources, monitors performance, and prevents deadlocks. The operating and accounting functions of the KPS are confined to the AM. The engineering and maintenance functions are also performed by the AM, such as updating of the KBs, altering the structure and hierarchy of the stored information in the KBs, and modifying the compiling and interpreting processes of the $\mathrm{CM}$ for its

6 Such as, "what," "how," “when," “where,” and "why”; and "what-if," "how-where," “what-when," “thenwhat" combinations; and also analogize, predict, and simulative combinations. 
many tasks. The knowledge rings (KRs) depicted in Figure 6 are organized according to the disciplines based on the Dewey Decimal System (DDS) or the Library of Congress (LoC) classification 7 .

\subsection{MULTIPROCESSOR-BASED KNOWLEDGE ENVIRONMENTS}

The administrative module (AM) that accomplishes the administrative functions, is also responsible for the execution of each subprocess in the problem solution or query handling by the

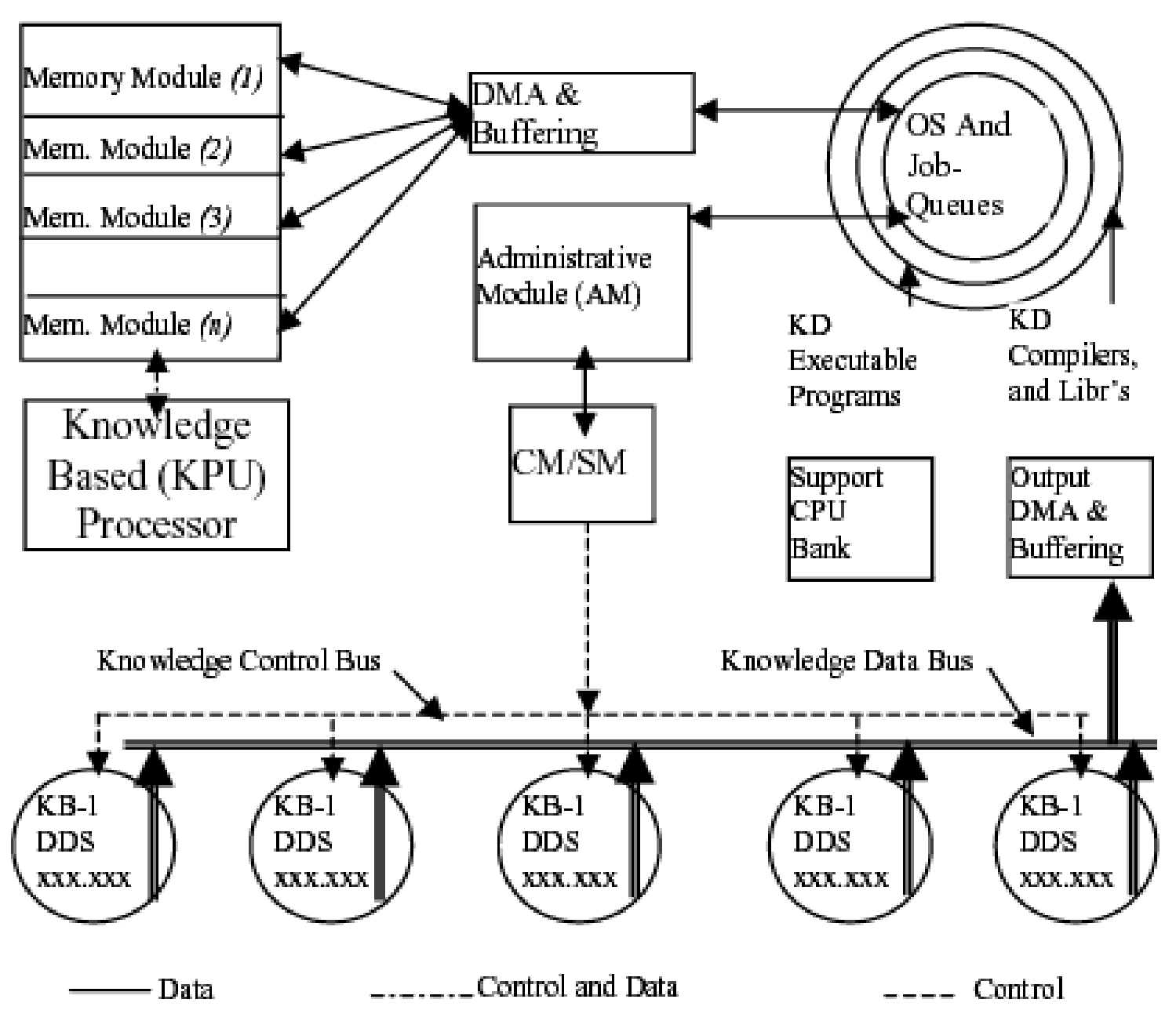

Figure 5 Architecture of a simple knowledge processing system with a single processor based on the electronic switching system (ESS) platform. CM, communication module; SM, switching module; KB, knowledge base.

\footnotetext{
7 To be consistent with the memory addresses in $2 \frac{1}{2} \mathrm{D}, 3 \mathrm{D}$, and multiple wafer memories, the numbering of the "knowledge rings" can also be binary. The need for external address translation is eliminated and the linkers/loaders can generate the executable code for any given machine. To some extent the scalability of the knowledge bases may be sacrificed because of the limited binary addresses available. This is truly a matter of detailed design of the entire knowledge processing system.
} 
KPS. In a sense, it becomes the hardware host for the KPS "operating system." We foresee that the AM is a parallel processor system, assuming the KPS is going to be shared between a large numbers of user tasks. Since any processor (in the AM) can access any information in the knowledge base, the switching module will switch between the processors of the AM and the $\mathrm{read} /$ write capability of the KBs. The quantity of information and its directionality may now be identified. In the great majority of cases, the KBs share large chunks of information with memory modules of the AM.

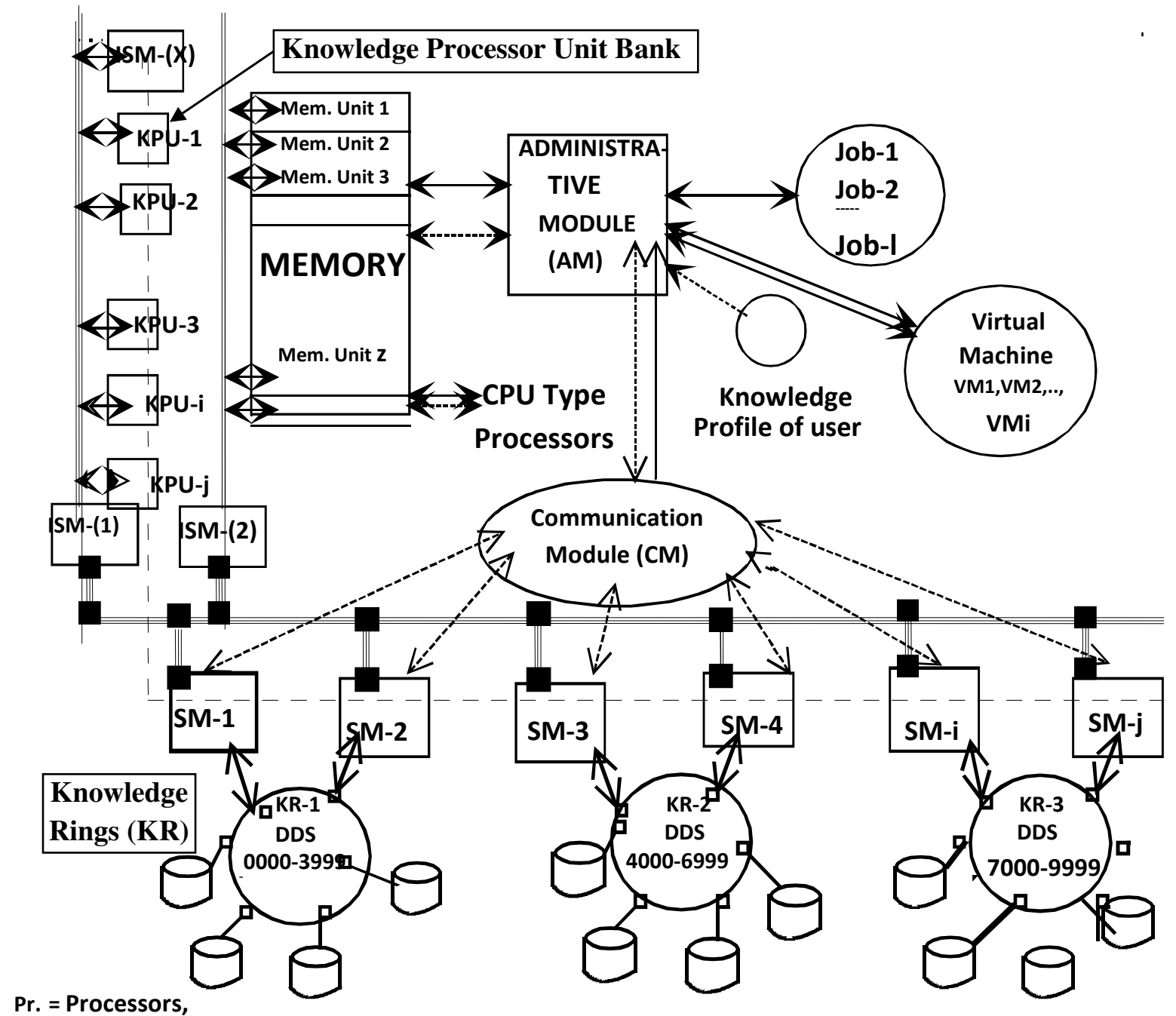

ISM(i) = Internal Switching Module, SM-i = Typical ESS Switching Module, KR = Knowledge Rings,

\section{- Control Signals, $\equiv$ Internal Bus Structures, DDS = Dewey Decimal/Library of Congress}

Figure 6 Typical architecture of the knowledge processing system with multiple processors, independent communication module (CM), and switching modules (SM). KPU=Knowledge processing unit, $\mathrm{CPU}=$ conventional central processor unit,

The "knowledge rings" are partitioned by the subject matter; however, any numbering or addressing system is appropriate. SM, switching module; KR, knowledge ring; KPS, knowledge processing systems or machines become essential. Secondary cache memories (not shown) can also 
be used to "fetch" object groups from the knowledge bases during processing. Detailed diagrams for parallel and pipeline types of knowledge processor are presented in [8]. After performing the knowledge functions (e.g., pattern recognition, matching, forward/backward pointing, and inference drawing), the contents of the memory may be discarded. Whereas the interrogation may be only one instruction long, the returned information can be a large block. Thus, the modality of communication between the AM and the KBs can be made asymmetrical, and for this reason a serial query port and DMA for burst-back appears to be a feasible implementation. The SM may also be designed for the low/high rates of data movement depending on the application.

In traditional electronic switching systems, the SM is located between the trunks (and subscriber links) from one geographic area to the next. In the KPS, the SM accesses the various processors (and memory modules) in the AM and the extensive fragments of knowledge in the KBs. The addressing in the KPS is done by the classification of knowledge and its hierarchy. Typically, the addressing is done via the area codes and exchange numbers. The address translation and seek time is dramatically reduced by the addressing of the subject material, on which the knowledge function is being performed, by its decimal code. The same code thus provides the exact address of that specific piece of knowledge in the KBs.

The bus capacity and memory allocation algorithms affect the performance. Since the amount of information necessary to perform knowledge functions can be vastly different depending on the topic and the type of operation, fixed memory allocation algorithms are soon likely to become constrictive. However, if the operating system has sufficient flexibility (such as dynamic memory allocation), the active memory size is made sufficient to handle complex KPS functions (such as, pattern recognition, cross-compare, find analogies, find supplementary knowledge, and scan other sciences). Then the switching module can provide access between all the AM processors and the file segments in the KBs in real time, and an architecture for intelligent knowledge processing may be generated.

The difficulties that we foresee in this methodology occur when all the users need or access the same information, for example, a class of students handling the solution to a given problem during the laboratory hour. Queue management techniques still lead to long response times. Another instance of such a situation arises if all the users are using memory-intensive subprocesses at the same time. Even though we have listed such functions as "bottlenecks," they are also encountered in all computer systems. Smart operating systems handle contingencies of this nature.

\section{CONCLUSIONS}

This paper presents two major aspects of knowledge. First, in dealing with knowledge, the truism tempered by long-term philosophic validation leads to scientific principles. These principles are formulated as qualitative and statistical relationships to start a basis of knowledge theory by which the differentiation, integration and sensitivity of knowledge can be estimated. Primary and secondary knowledge objects are introduced to offer knowledge structure and dependence. The quantitative basis and content of a body of knowledge are established by the number of secondary objects, their structural relationships, the number of attributes of each secondary object, and their own relation matrices.

Granularity of the knowledge space is defined as the smallest prism formed by the numerical precision of the computer systems, the lowest Hamming distance between the code words that the networks can carry at their maximum speed, and the perception of human beings who will sense the microprism of knowledge. At least one dimension of this prism is personality dependent, even 
though the numerical precision of the computers and lowest Hamming distance through the network can be accurately quantified for that particular human-machine system.

Second, in dealing with the theory of knowledge, the comparison with complexity theory shows that knowledge theory is closely intertwined to the quantity of knowledge (see the paragraph above) in any primary knowledge object. However, knowledge theory is always retractable and (almost) never gets chaotic for three reasons.

(1) The linkage (forward and backward pointers, depth of recursion, size of memory) built in the operating systems of computers will prevent tail-chasing loops through the many knowledge objects.

(2) The seven OSI layers will automatically prevent networks from getting trapped in endless sendresend cycles of any packets, sessions, blocks, knowledge objects and so on.

(3) The human beings who monitor the machines are capable of preventing machines from senseless and silly pursuits in the knowledge domain.

Knowledge processing systems are based firmly on the triad of machines, networks and humans working in conjunction and cooperation. Three mechanisms \{ the machines, (their architectures and operating systems), the networks (their layering and protocol) and the human beings (their natural intelligentsia)\} work synergistically in making knowledge environment complex but manageable.

\section{REFERENCES}

1. J. M. Keynes, The General Theory of Employment, Interest, and Money, Prometheus Books; Buffalo, NY, 1997.

2. J. Neumann and O. Morgenstern, Theory of Games and Economic Behavior, Princeton University Press, Princeton, NJ, 2004.

3. J. R. Parker, Algorithms for Image Processing and Computer Vision, John Wiley and Sons, Hoboken, NJ, 1996.

4. R. Arena and M. Quere (Eds) The Economics of Alfred Marshall: Revisiting Marshall's Legacy, Palgrave Macmillan, New York, 2003.

5. A. Smith, The Wealth of Nations, Prometheus Books, Buffalo, NY, 1991.

6. D. S. Byrne Complexity Theory and Social Sciences, Routledge, New York, 1998.

7. S. V. Ahamed, Intelligent Internet Knowledge Networks, John Wiley and Sons, Hoboken, New Jersey 2006.

8. S. V. Ahamed, Computational Framework of Knowledege, Chapter 7, John Wiley and Sons, Hoboken, New Jersey 2009.

9. OCLC, Dewey Decimal Classification and Relative Index, 22nd ed., OCLC, Dublin, OH, 2003. See also, J. P. Comaroni, Dewey Decimal Classification, 18th ed., Forest Press, Albany, NY, 1976.

10. United States Government, Library of Congress Classification, <http://catalog.loc.gov> URL accessed June 2003. 